\section{Nonmetric multidimensional scaling of differences among type*}

\author{
AKINORI OKADAT, Chiba University, Chiba-ken, Japan \\ and \\ YOSHIKO TAKEUCHI, Keio University, Tokyo, Japan
}

Sixteen Ss analyzed differences among eight kinds of type styles by means of a nonmetric multidimensional scaling procedure. This procedure is a means of explaining individual differences in perceptual structure and gives us a perceptual structure for each of the Ss as well as for a configuration of Ss. Ss discriminated differences in the color density (blackishness) and in the actual styles of the type.

The purpose of this study was to determine if perceived differences among various kinds of type styles could be represented adequately by distance in real Euclidean space by means of a multidimensional scaling. The multidimensional scaling procedure used in this study was a nonmetric one, such as Tucker \& Messick's (1963), that investigated individual differences in perceptual structure.

\section{STIMULI}

Eight kinds of type styles were selected, and a line of capital letters of the English alphabet (ABC ...Z Z) of each style was used as a stimulus. These were (1) Palace Script, (2) Gill Sans Medium, (3) Century Old Style Italics, (4) Alternate Gothic, (5) Century old Style, (6) Wedding Text, (7) Bernhard Modern, and (8) Park Avenue, all 12-point and adequately spaced. They are represented in Fig. 1. Twenty-eight pairs were formed by combining any two styles. Each pair was printed in the center of a thick white $10 \times 15 \mathrm{~cm}$ cardboard so that two types were parallel with each other and with the longer side of the cardboard. The interval between the upper alphabetical line and the lower alphabetical line was $1.1 \mathrm{~cm}$, and the two alphabetical lines were printed so that the middle points of each alphabetical line were on the vertical center line of the cardboard.

\section{SUBJECTS}

Sixteen Ss participated in the experiment. Ss 1, 2, and 3 were students in electrical engineering; Ss 4 , $5,6,7,8,9,10,11$, and 12 were in

*This research was supported by the Institute of Information Science and the Department of Administrative Engineering. both of Keio University. The authors wish to thank Drs. Tarow Indow and Shoji Ura for their helpful suggestions and Miss Keiko Matsushima for her help in executing the experiment.

†Address: 2531-3 Ozenji. Kawasaki-shi, Kanagawa-ken. Japan. administrative engineering; Ss 13, 14, 15 , and 16 were in psychology. Ss 9 , $13,14,15$, and 16 were female. None of them had special knowledge about type styles or typography. All Ss except S 15 had served in at least one preliminary experiment using type styles, some of which had been the same as those used in this experiment.

\section{PROCEDURE}

Each $\mathrm{S}$ was provided with 28 cards on which a stimulus pair had been printed and was told to arrange them on a table covered with white paper in ascending order according to perceived dissimilarities between the two alphabetical lines on each card. Ranks given to each of the 28 pairs were recorded for each $\mathrm{S}$.

\section{MULTIDIMENSIONAL SCALING PROCEDURE}

The perceptual structure of each $S$ was obtained by Kruskal's method $(1964 a, b)$ from the ranks given to 28 interstimulus dissimilarities. Then to obtain the configuration of Ss which described relationships among their judgments of 28 interstimulus dissimilarities, we obtained dissimilarities between Ss' ranks applied to the interstimulus dissimilarities. Dissimilarity between

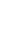

jth S's judgment for interstimulus dissimilarities and $\mathrm{k}^{\text {th }}$ S's judgment in the present study was defined by

$$
\delta_{j k}=1-r_{j k},
$$

where $r_{j k}$ is a Kendall rank correlation coefficient between ranks applied to interstimulus dissimilarities given by $j^{\text {th }} \mathrm{S}$ and by $k^{\text {th }} \mathrm{S}$. A similar formula is shown in Shepard's paper (1962, p. 241) in connection with discussion concerning the relationship between factor analysis and his analysis of proximities. The main point of the present multidimensional scaling procedure is that it is nonmetric. Namely, each of the perceptual structures and the configuration of Ss can be derived only from ordinal relationships among interstimulus dissimilarities.

RESULTS AND DISCUSSION

To obtain the perceptual structure of each of the $16 \mathrm{Ss}$, separate multidimensional scaling was done on each set of ranks given by each $S$ by means of Kruskal's method (1964a, b) in uni- and two-dimensional spaces for the Minkovski $r$ of 2. Because of the ease of interpretation of perceptual structures (Kruskal, 1964a, p. 16), the perceptual structure of each of the 16 Ss was determined to be unidimensional, except that of $\mathrm{S} 10$, whose perceptual structure was two-dimensional. The perceptual structure of each of the $16 \mathrm{Ss}$ and the associated stresses are shown in Fig. 2. The configuration of $S s$ was also derived by Kruskal's method in uniand two-dimensional spaces for the Minkovski r of 2, using dissimilarities calculated by (1). The lowest stress yielded in unidimensional space was 0.233 ; the lowest stress yielded in two-dimensional space was 0.102 , and in this case, the dimensionality was determined to be 1 . The configurations of the 16 Ss are shown in Fig. 3. Stress of the configuration of the Ss and some of the stresses shown

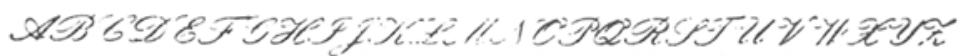
ABCDEFGHIJKLMNOPQRSTUVWXYZ ABCDEFGHIJKL.MNOPQRSTUVWXYZ
ABCDEFGHIJKLMNOPQRSTUVWXYZ ABCDEFGHIJKLMINOPQRSTLVWXYZ

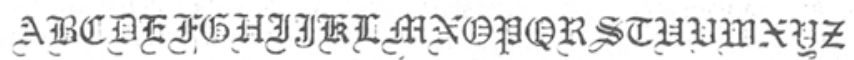
ABCDEFGHIRLMNOPQRETLVWXYZ

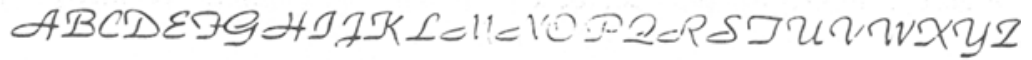

Fig. 1. Eight kinds of types. 
SUBJEC

\section{STRESS 234576}

$0001 \longrightarrow x_{6}$

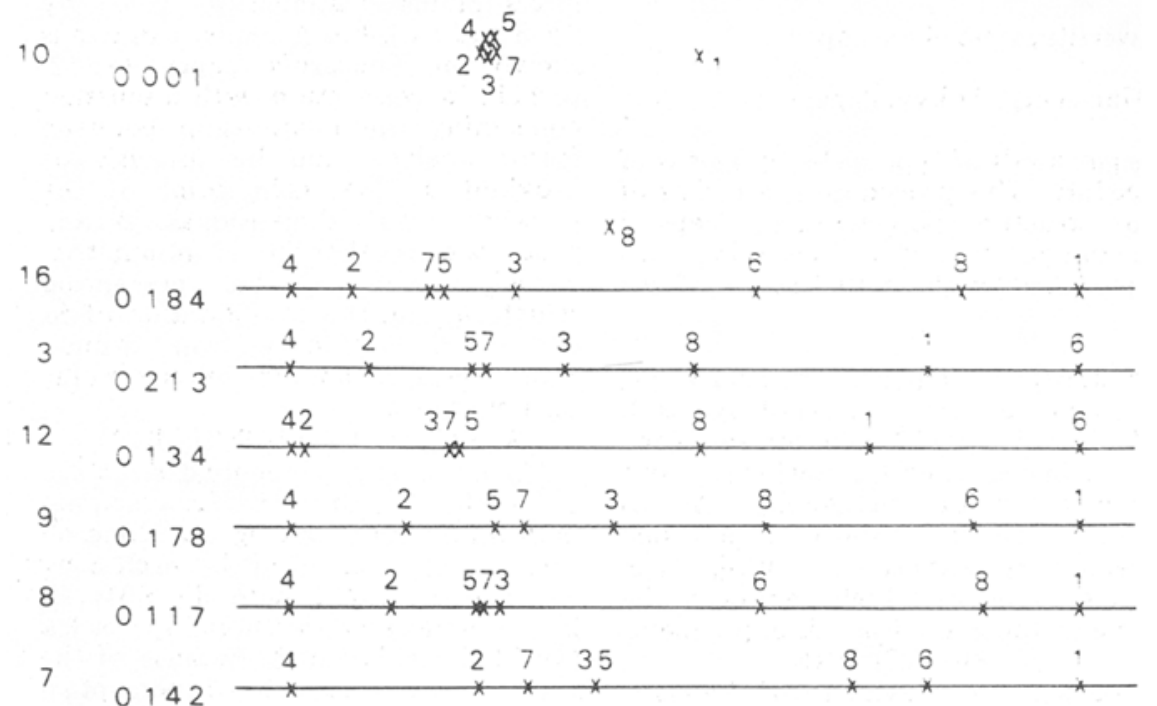

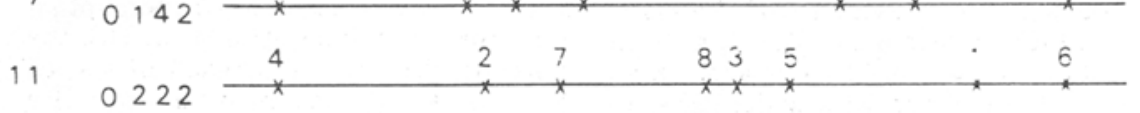

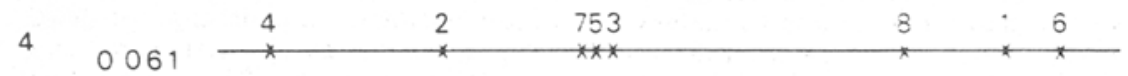

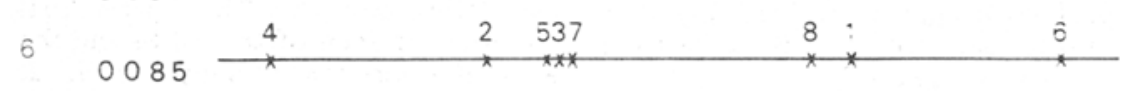

$130175 \begin{array}{llllll}4 & 2 & 5 & 7 & 3 & 8\end{array}$

$20088 \begin{array}{lllllll}4 & 2 & 3 & 5 & 7 & 6 & 18 \\ & x & & \end{array}$

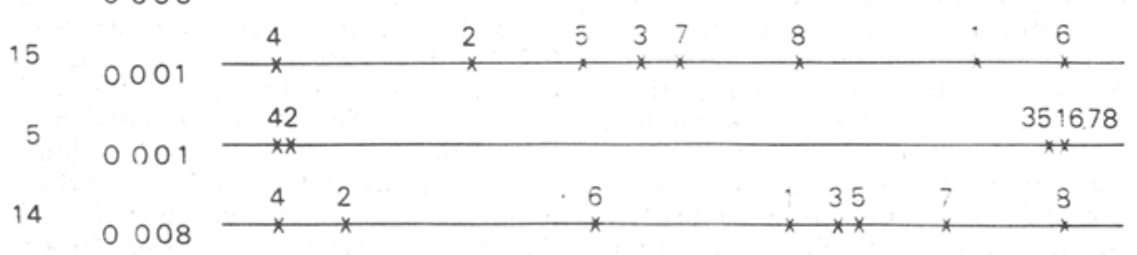

Fig. 2. Perceptual structure of each S.

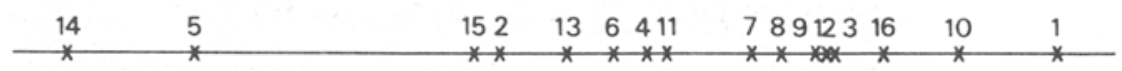

Fig. 3. Configuration of Ss.

in Fig. 2 seem to be somewhat too large compared with the verbal evaluation suggested by Kruskal (1964a, p. 3), but they are acceptable according to a recent paper by Klahr (1969).

Figure 2 represents the perceptual structure of each of the $16 \mathrm{Ss}$ in the same order of the corresponding $\mathrm{Ss}$ in Fig. 3. We can see from Figs. 2 and 3 that in the perceptual structures of Ss 14 and 5 (see left end of Fig. 3), Stimuli 2 and 4 are isolated from the remaining stimuli. Similarly, in the perceptual structures of Ss 1,10 , and 16 (right end in Fig. 3), Stimuli 1 and 8 , or Stimuli 1, 6, and 8, are isolated from the remaining stimuli. In the perceptual structures of Ss $3,12,9,8$, and 7 , the tendency of Stimuli 1,6 , and 8 to be isolated from the remaining stimuli is recognized, but it is not so marked as in the case of Ss 1 , 10 , and 16 . In the perceptual structures of Ss $11,4,6,13,2$, and 15 , the groups of Stimuli 1, 6, 8 and of Stimuli 2,4 are both isolated from Stimuli 3,5 , and 7 . This tendency is also found slightly in the perceptual structures of Ss $3,12,9,8$, and 7 .

Stimuli 2 and 4 are characterized by their blackishness and Stimuli 1, 6, and 8 , by their distinctive style. Stimuli 3,5 , and 7 are characterized neither by blackishness nor by distinctive style. Thus we can say that Ss located at the left side in Fig. 3 consider the difference in blackishness of types important, that Ss located at the right side in Fig. 3 consider important the difference in style of types, and that Ss located at the center in Fig. 3 consider differences both in blackishness and style of types significant when they judge interstimulus dissimilarities. Thus the reference axis in Fig. 3 represents the importance Ss give to blackishness or to style.

As for attributes of type styles, we can enumerate blackishness, style, presence or absence of serif, form of serif, italic or not italic, and condensed type or expanded type. As far as the present experiment is concerned, each $\mathbf{S}$ paid attention to the differences in blackishness and style, but the differences in the remaining attributes were neglected. If more assertive conclusions about attributes explaining differences among various kinds of type styles are expected, experiments including more kinds of type styles and more Ss will be needed.

\section{REFERENCES}

KLAHR, D. A Monte Carlo investigation of the statistical significance of Kruskal's nonmetric scaling procedure. Psychometrika, 1969, 34, 319-330.

KRUSKAL, J. B. Multidimensional scaling by optimizing goodness of fit to a nonmetric hypothesis. Psychometrika, 1964a, 29, 1-27.

KRUSKAL, J. B. Nonmetric multidimensional scaling: A numerical method. Psychometrika, 1964b, 29. 115-129.

SHEPARD, R, N. The analysis of proximities: Multidimensional scaling with an unk nown distance function. II. Psychometrik a, 1962, 27, 219-246.

TUCKER, L. R. \& MESSICK, S. An individual differences model for multidimensional scaling. Psychometrik a, 1963, 28, 333-367. 